\title{
An Adaptive PMU-Based Wide Area Backup Protection Scheme for Power Transmission Lines
}

\author{
M. Kalantar Neyestanaki, Student Member, IEEE, and A. M. Ranjbar
}

\begin{abstract}
This paper proposes a novel adaptive wide area backup protection scheme for transmission lines. In the proposed scheme, both the faulted line and fault location are determined by a limited number of synchronized phasor measurements. Based on phasor measurement unit placement and network topology, subsets of lines and buses called backup protection zones (BPZs) are formed. After a fault occurs in the transmission network, the sum of zero- and/or positive-sequence currents entering the faulted BPZ highly increases, and hence, the faulted BPZ can be determined. The linear least squares method is then used to determine the faulted line, as well as the fault location by voltage and current phasors of the faulted BPZ. Accordingly, the proposed scheme provides a closed-form and noniterative solution for the faulted line and fault location identification problem. On the other hand, it readily determines the faulted line regardless of the fault type, fault resistance, and measurement errors. To show the effectiveness of the method, it is applied to the WSCC 9-bus and IEEE 118-bus test systems. Simulation results verify successful identification of the faulted BPZ as well as the faulted line within the faulted BPZ with limited measurement points.
\end{abstract}

Index Terms-Backup protection zone (BPZ), phasor measurement unit (PMU), smart transmission grid, wide area backup protection, wide area measurement system (WAMS).

\section{NOMENCLATURE}

$a_{i j}$

$B$

$d_{0, \mathrm{BPZ}}$

$I_{t h, \mathrm{BPZ}}^{(0)}$

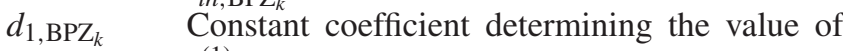
$I_{t h, \mathrm{BPZ}_{k}}^{(1)}$.

$e_{j} \quad$ Binary parameter that is equal to 1 if bus $j$ is a zero injection bus and 0 otherwise.

$f \quad$ Fictitious bus at the fault point.

$f_{1}, f_{2} \quad$ Two end buses of the faulted line.

$G \quad$ Set of generator buses.

$H \quad\left(n_{p}+n_{L}\right)$ by two matrix containing the constant coefficients.

$h_{1}, h_{2} \quad$ First and second columns of matrix $\mathrm{H}$, respectively.

Manuscript received July 21, 2014; revised November 8, 2014 and December 22, 2014; accepted December 23, 2014. Date of publication January 20, 2015; date of current version April 17, 2015. Paper no. TSG-00747-2014.

The authors are with the Center of Excellence in Power System Management and Control, Department of Electrical Engineering, Sharif University of Technology, Tehran 11365-11155, Iran (e-mail: kalantar@ee.sharif.edu).

Digital Object Identifier 10.1109/TSG.2014.2387392 $i, j, r, l_{1}, l_{2}$ Indices of bus.

$I_{\text {ent }, i} \quad$ Total current entering the faulted backup protection zone (BPZ) through the $i$ th phasor measurement unit (PMU) bus.

$I_{\text {ent, BPZ }}^{(s)} \quad$ Magnitude of the sum of $s$-sequence currents entering the $k$ th BPZ.

$\overline{I_{\mathrm{ent}, \mathrm{BPZ}}^{(1)}} \quad$ Average of $I_{\mathrm{ent}, \mathrm{BPZ}}^{(1)}$ in the last minute.

$I_{f}$

$I_{l}^{(s)}$

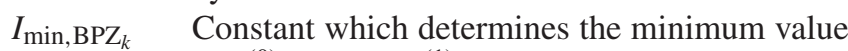
of $I_{t h, \mathrm{BPZ}_{k}}^{(0)}$ and $I_{t h, \mathrm{BPZ}}^{(1)}$.

$I_{t h, \mathrm{BPZ}_{k}}^{(s)} \quad$ Threshold of $I_{\mathrm{ent}, \mathrm{BPZ}_{k}}^{(s)}$.

$J(u) \quad$ Relative residual error of estimation.

$k \quad$ Index of BPZ.

$l \quad$ Index of line.

$L_{k} \quad$ Set of lines that belong to the $k$ th BPZ and are connected to the surrounding PMU buses.

Measurement Vector.

$m$

Total number of buses of the faulted BPZ (equal to $n_{p}+n_{n p}$ ).

Number of transmission lines that belong to the faulted BPZ and are connected to the surrounding PMU buses.

Number of non-PMU buses of the faulted BPZ. Number of PMU buses of the faulted BPZ.

Observability function of bus $i$.

Binary decision variable that is equal to 1 if a PMU is installed at bus $i$ and 0 otherwise.

$\begin{array}{ll}u & \text { Unknown variable vector. } \\ V_{i} & \text { Positive-sequence voltage of bus } i \text { which is }\end{array}$ measured by a PMU.

$w_{i j} \quad$ Auxiliary binary variable of buses $i$ and $j$, which models the effect of zero injection buses.

$x \quad$ Per-unit distance between fault point $f$ and bus $f_{1}$.

$y_{l} \quad$ Ground admittance of line $l$.

$z_{l} \quad$ Line impedance of line $l$.

$Z \quad$ Bus impedance matrix of the faulted BPZ including fictitious bus $f$.

$Z^{0} \quad$ Pre-fault bus impedance matrix of the faulted BPZ excluding fictitious bus $f$. 


\section{INTRODUCTION}

$\mathbf{T}$ RANSMISSION lines are protected by primary and backup protection systems. Traditional backup protection systems basically include earth fault overcurrent and overreaching zones of distance relays [1], [2]. These relays are self-contained, use only local measurements, do not adapt their characteristics to the power system conditions, do not communicate together, do not consider the infeed and outfeed effects and do not consider the fault resistance, conventionally [3], [4].

In modern societies, power networks are widely speared over large geographical areas, transmission grids are more interconnected, and transmission lines are utilized close to their limits. Hence, the traditional backup protection systems can misoperate when the power system is extremely under stress and the transmission lines are operated close to their limits, while in these situations the hazard of cascading events is considerable [5]. For example, the unwanted operation of the third zone of distance relays has been recognized as one of the most important contributors to cascading outages, which lead to major power system blackouts [6]. Therefore, there is a growing need for novel backup protection schemes to improve power system security.

Recent advances in solid-state technology, communication systems, and distributed computing have resulted in significant developments in modern sensors [3]. One of these sensors is PMU, which provides the synchrophasors of voltage and current by using global positioning system (GPS) signals. Wide area measurement system (WAMS) is implemented by widespread installation of PMUs in a power system and uses high-speed communication systems to gather synchronized phasor measurements. WAMS technology facilitates wide area applications, such as state estimation, oscillation damping, remedial action schemes and special protection schemes [7]-[10].

The problems of traditional backup protection relays can be resolved by using novel backup protection schemes, which utilize phasor measurements obtained from WAMS. In this area, current differential schemes are proposed in [11] and [12]. These differential schemes provide backup protection of a line by using GPS-synchronized current measurements at both ends of the line. Jiang et al. [13] introduces an adaptive PMUbased protection scheme for a transmission line. This scheme uses Clarke components of synchronized voltage and current phasors at two ends of the line. A wide area backup protection algorithm for transmission lines is presented in [14]. The algorithm compares the positive-sequence voltage magnitude of buses to detect the nearest bus to the fault. Next, the faulted line is detected by comparing the absolute differences of positive-sequence current angles for all lines connected to the selected bus. The above algorithms can be implemented for the whole transmission network, if and only if, all buses of the power network are provided by PMUs. Ma et al. [15] proposed a fault steady state component-based wide area backup protection algorithm. This algorithm utilizes the bus impedance matrix of the faulted region to distinguish the faulted branch by using the fault steady state component of the currents and voltages. This method, however, requires all non-zero injection buses to be equipped with PMU. Liao [16] proposes a fault location method based on sparse measurements, even if the measurements are not from the faulted line, this method precisely determines the fault location.

In this paper, a novel adaptive wide area backup protection scheme for transmission lines is developed to identify the faulted line by using the wide area data of WAMS. First, an optimization model is developed to minimize the number of PMUs required for this scheme. This overcomes the problems of data storage limitations and requirements of extensive communication facilities and infrastructure. Next, the proposed backup protection scheme is introduced. After a fault occurs on a transmission line, this scheme first determines the faulted BPZ and next determines the faulted line in the faulted BPZ. If the primary protection system fails and the faulted line is not isolated, this backup protection scheme take over and isolates the faulted line. Since the proposed scheme operates based on the wide area data of WAMS, it is efficient even if the faulted line is not equipped with any PMUs. Moreover, this scheme provides a closed-form and non-iterative solution for the faulted line and fault location identification problem by utilizing the linear least squares method. In other words, this scheme has a very low computation burden and so this scheme quickly identifies the faulted line and fault location. Accordingly, this scheme minimizes the impact of faults on the power system by quickly and precisely determining the faulted line. Therefore, this scheme improves the security of power system.

The organization of this paper is as follows. In Section II, rules of PMU placement and BPZ formation are described. Section III describes the algorithm for faulted zone identification. In Section IV, an algorithm based on the linear least squares method is proposed for distinguishing the faulted line. Simulation results to prove the effectiveness of the method are demonstrated in Section V.

\section{Formation of Backup Protection Zone}

\section{A. PMU Placement for Backup Protection}

The proposed wide area backup protection scheme detects the faulted zone and then determines the faulted line by utilizing the measurement data of WAMS and bus impedance matrix. In other words, this scheme is independent from generators model and parameters. Therefore, this scheme requires that the generator buses are equipped with PMUs. But so far, this constraint has not been considered in the optimal PMU placement problem. Hence, in order to implement this application in smart transmission grids, this constraint must be considered in the optimal PMU placement problem. Accordingly, the optimal PMU placement problem is formulated as follows:

$$
\begin{aligned}
\text { Minimize } \sum_{i \in B} p_{i} & \\
\text { Subject to } \quad O_{i} \geq 1, & \forall i \in B \\
p_{i}=1, & \forall i \in G .
\end{aligned}
$$

In (2), $O_{i}$ is the observability function of bus $i . O_{i} \geq 1$ indicates that the $i$ th bus is observable. 


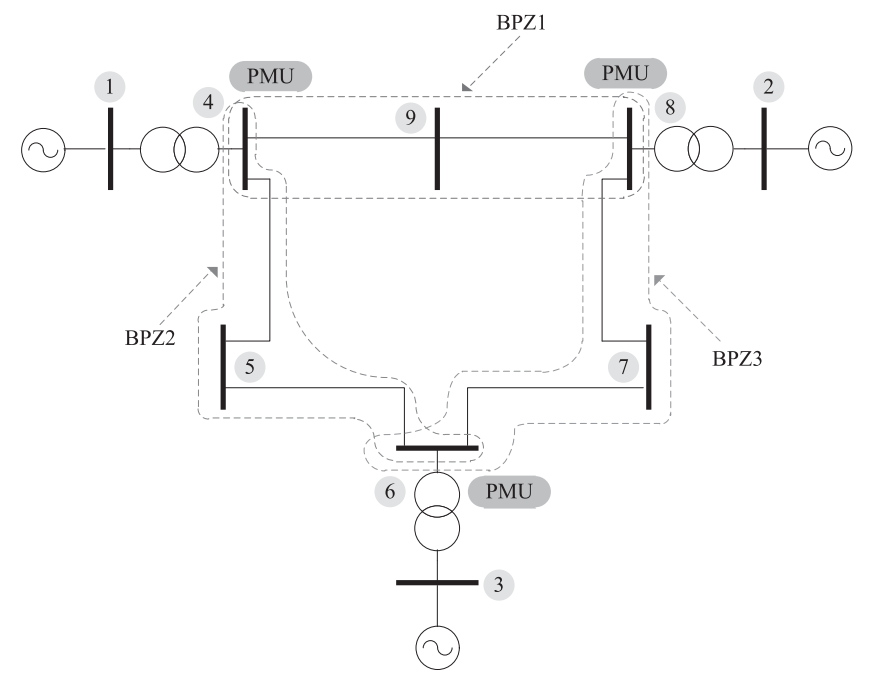

Fig. 1. Formation of back-up protection zones for the WSCC 9-bus test system.

A bus is called a zero injection bus, if it has no load or generator connected to it. In other words, no current is injected to the zero injection bus. For a zero injection bus and its adjoining buses, if all these buses are observable except one, the unobservable bus will also be observable by using the KCL at the zero injection bus. The effect of zero injection buses must be considered in the optimal PMU placement and thus the observability function of bus $i$ is as follows [17], [18]:

$$
\begin{array}{ll}
O_{i}=\sum_{j \in B} a_{i j} p_{j}+\sum_{j \in B} a_{i j} e_{j} w_{i j}, & \forall i \in B \\
\sum_{i \in B} a_{i j} w_{i j}=e_{j}, & \forall j \in B .
\end{array}
$$

In the above equations, $a_{i j}$ is a binary connectivity parameter defined as

$$
a_{i j}= \begin{cases}1, & \text { if } i=j \\ 1, & \text { if buses } i \text { and } j \text { are connected } \\ 0, & \text { otherwise. }\end{cases}
$$

The solution of the optimal PMU placement problem is obtained by solving the linear objective function (1) subject to the constraints (2)-(5). The measurement limitations can be added to the optimal PMU placement problem for backup protection, similar to the proposed method in [17].

\section{B. Formation of Backup Protection Zone}

BPZs are formed on the basis of the PMU placement and network topology. Each BPZ consists of the lines and buses that are surrounded by PMU-equipped buses. The proposed algorithm for optimal PMU placement and formation of BPZs is applied to the WSCC 9-bus test system. This system along with associated BPZs is shown in Fig. 1.

\section{FAULTED Zone IdENTIFICATION ALGORITHM}

As mentioned in the previous section, each BPZ is surrounded by PMUs. Hence, all currents, which enter each BPZ, are measured by corresponding PMUs. Also, if a fault occurs in the $k$ th BPZ, the sum of zero- and/or positive-sequence currents entering this BPZ increases and will be significantly greater than that of pre-fault conditions. Therefore, if the magnitude of the sum of zero- or positive-sequence currents entering the $k$ th BPZ is greater than the related threshold of this BPZ, it is specified as the faulted zone. In other words, the $k$ th BPZ is selected as the faulted BPZ, if the following equation is satisfied for either zero- or positive-sequence in this BPZ:

$$
I_{\mathrm{ent}, \mathrm{BPZ}}^{(s)}>I_{t h, \mathrm{BPZ}}^{(s)}
$$

where

$$
I_{\mathrm{ent}, \mathrm{BPZ}}^{(s)}=\left|\sum_{l \in L_{k}} I_{l}^{(s)}\right| .
$$

By monitoring the per-fault currents entering the $k$ th BPZ, the thresholds of this BPZ are defined in an adaptive manner, as follows:

$$
\begin{aligned}
& I_{t h, \mathrm{BPZ}_{k}}^{(0)}=\max \left\{d_{0, \mathrm{BPZ}_{k}} \overline{I_{\mathrm{ent}, \mathrm{BPZ}}^{(1)}}, I_{\min , \mathrm{BPZ}_{k}}\right\} \\
& I_{t h, \mathrm{BPZ}_{k}}^{(1)}=\max \left\{d_{1, \mathrm{BPZ}} \overline{I_{\mathrm{ent}, \mathrm{BPZ}}^{(1)}}, I_{\min , \mathrm{BPZ}_{k}}\right\}
\end{aligned}
$$

where the superscripts (0) and (1) denote the zero- and positive-sequence measurements, respectively. $I_{\min , \mathrm{BPZ}_{k}}$ is a constant which determines the minimum value of $I_{t h, \mathrm{BPZ}_{k}}^{(s)}$ and takes a value between 0 to 1 p.u. For the BPZs having no loads, $I_{t h, \mathrm{BPZ}_{k}}^{(s)}$ will be equal to $I_{\min , \mathrm{BPZ}_{k}} \cdot I_{\min , \mathrm{BPZ}_{k}}$ is determined based on power system transient studies. At each time, $\overline{I_{\mathrm{ent}, \mathrm{BPZ}}^{(1)}}$ is the average of $I_{\mathrm{ent}, \mathrm{BPZ}}^{(1)}$ in the last minute. $d_{0, \mathrm{BPZ}}$ and $d_{1, \mathrm{BPZ}}$ are constant coefficients determining $I_{t h, \mathrm{BPZ}_{k}}^{(0)}$ and $I_{t h, \mathrm{BPZ}_{k}}^{(1)}$, respectively. This algorithm has been inspired by the directional overcurrent protection scheme; $I_{t h, \mathrm{BPZ}_{k}}^{(s)}$ plays the same role here as does the pickup current in directional overcurrent relays. Therefore, $d_{0, \mathrm{BPZ}}$ and $d_{1, \mathrm{BPZ}_{k}}$ are determined in a way similar way to the pickup current of ground and phase directional overcurrent relays, respectively. In other words, the value of $d_{0, \mathrm{BPZ}_{k}}$ is greater than 0 and less than 1 and the value of $d_{1, \mathrm{BPZ}}$ is greater than 1 and less than 2 . The pickup setting of phase and ground directional overcurrent relays comprehensively have been discussed in [2].

According to (9) and (10), by updating $\overline{I_{\mathrm{ent}}^{(1)} \mathrm{BPZ}_{k}}$, the thresholds of the $k$ th BPZ are adapted to the power system conditions, hence, the security and dependability of the proposed fault zone identification algorithm are both guaranteed.

If the generator buses are not equipped with PMUs, they are not possible to be separated from the BPZs and then, some BPZs would involve one or more generators. If a fault occurs in one of such BPZs, the proposed fault zone identification algorithm might not detect the fault occurrence, since the fault current would be also supplied by the generators located in the faulted BPZ. Therefore, in order to implement the proposed fault zone identification algorithm in smart transmission grids, the generator buses must be equipped with PMUs. 


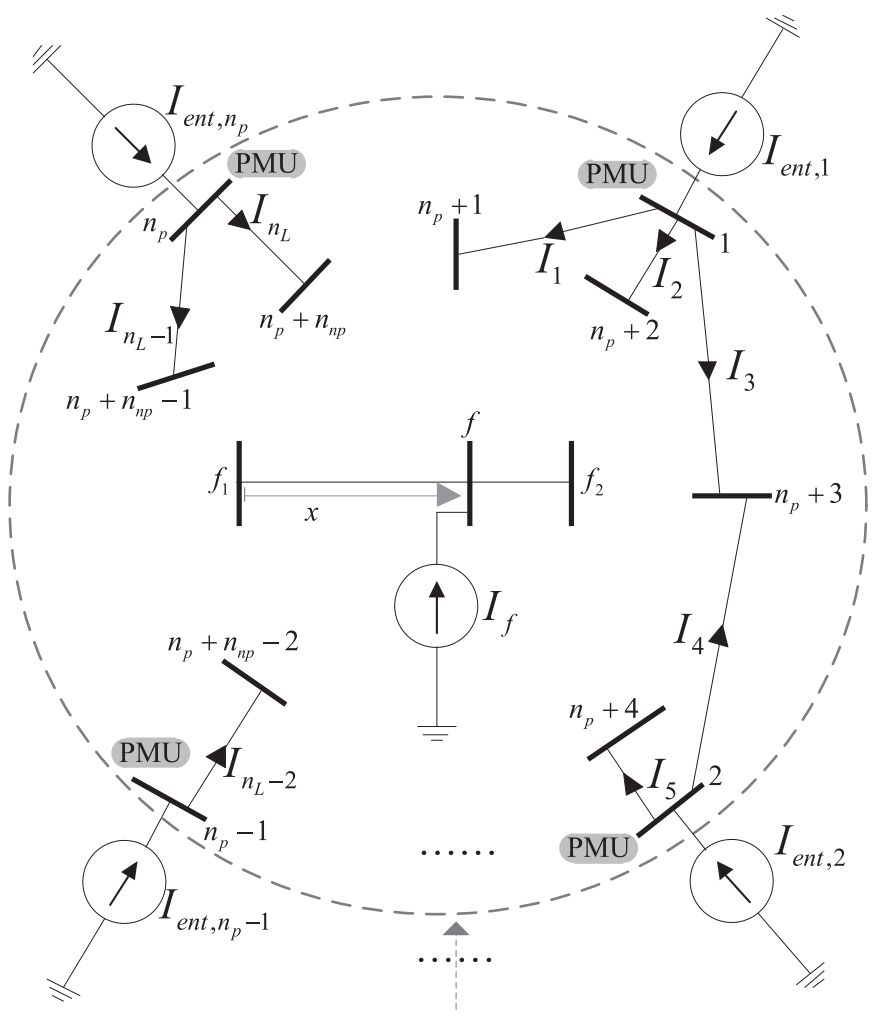

Faulted BPZ

Fig. 2. Identified faulted BPZ.

\section{FAulted Line Identification Algorithm}

The goal of this algorithm is identification of the faulted line in the faulted BPZ. According to the proposed method for PMU placement and BPZ formation, all boundary buses of the faulted BPZ are equipped with PMUs and the internal buses have no PMU. A faulted BPZ containing $n_{p}$ PMU buses and $n_{n p}$ non-PMU buses is shown in Fig. 2.

As shown in Fig. 2, the faulted BPZ can be considered as a sub-network which is connected to the remaining network by surrounding PMU buses. The remaining network is modeled by equivalent current sources. In this figure, $I_{\mathrm{ent}, i}$ is the total current entering the faulted BPZ through the $i$ th PMU bus. $f_{1}$ and $f_{2}$ are two end buses of the faulted line; $f$ is a fictitious bus at the fault point; $x$ is per-unit distance between fault point $f$ and bus $f_{1}$; and $I_{f}$ is the fault current injected into the fault point.

In Fig. 2, boundary PMU buses are connected to some non-PMU buses via $n_{L}$ transmission lines. Currents entering from PMU buses to these lines are $I_{1}, I_{2}, \ldots, I_{n_{L}}$. Line impedance and ground admittance of these lines are respectively, $z_{l}$ and $y_{l}$ where $l=1, \ldots, n_{L} . V_{1}, V_{2}, \ldots, V_{n_{p}}$ are voltages of the $n_{p}$ boundary PMU buses.

Only voltage and current phasors measured by boundary PMUs are available for identification of the faulted line in the faulted BPZ. Voltages of boundary buses, which are measured by PMUs, can be expressed as [19]

$$
V_{i}=Z_{i f} I_{f}+\sum_{r=1}^{n_{p}} Z_{i r} I_{\mathrm{ent}, r} \forall i=1,2, \ldots, n_{p}
$$

where $Z_{i, j}$ is the $i-j$ element of the matrix $Z . Z$ is the bus impedance matrix of the faulted BPZ including fictitious bus $f$, i.e., the dimension of $Z$ is $\left(n_{b}+1\right) \times\left(n_{b}+1\right)$, where $n_{b}=n_{p}+n_{n p}$. The elements of $Z$ can be calculated by utilizing the elements of $Z^{0} . Z^{0}$ is the pre-fault bus impedance matrix of the faulted BPZ, i.e., this matrix does not include the fictitious bus $f$, and hence its dimension is $n_{b} \times n_{b}$. Matrix $Z^{0}$ can easily be constituted [20]. The elements of this matrix are as follows:

$$
Z^{0}=\left[\begin{array}{ccrrrrr}
Z_{11}^{0} & \cdots & Z_{1 f_{1}}^{0} & \cdots & Z_{1 f_{2}}^{0} & \cdots & Z_{1 n_{b}}^{0} \\
\vdots & & \vdots & & \vdots & & \vdots \\
Z_{f_{1} 1}^{0} & \cdots & Z_{f_{1} f_{1}}^{0} & \cdots & Z_{f_{1} f_{2}}^{0} & \cdots & Z_{f_{1} n_{b}}^{0} \\
\vdots & & \vdots & & \vdots & & \vdots \\
Z_{f_{2} 1}^{0} & \cdots & Z_{f_{2} f_{1}}^{0} & \cdots & Z_{f_{2} f_{2}}^{0} & \cdots & Z_{f_{2} n_{b}}^{0} \\
\vdots & & \vdots & & \vdots & & \vdots \\
Z_{n_{b} 1}^{0} & \cdots & Z_{n_{b} f_{1}}^{0} & \cdots & Z_{n_{b} f_{2}}^{0} & \cdots & Z_{n_{b} n_{b}}^{0}
\end{array}\right] .
$$

$Z_{i r}$ is determined by utilizing the elements of $Z^{0}$, as follows [20]:

$$
Z_{i r}=Z_{i r}^{0} \quad \forall i, r=1, \ldots, n_{b}
$$

and by neglecting the shunt capacitance of the faulted line, $Z_{i f}$ can be determined as [16]

$$
Z_{i f}=Z_{i f_{1}}^{0}+\left(Z_{i f_{2}}^{0}-Z_{i f_{1}}^{0}\right) x \quad \forall i=1, \ldots, n_{b} .
$$

The currents of transmission lines which are connected to the PMU buses are measured by PMUs. According to the KCL, these currents can be expressed as follows [19], [21]:

$$
I_{l}=\frac{V_{l_{1}}-V_{l_{2}}}{z_{l}}+\frac{y_{l}}{2} V_{l_{1}} \quad \forall l=1, \ldots, n_{L}
$$

where $l$ is the line that connects PMU bus $l_{1}$ to the non-PMU bus $l_{2}$. By substituting (11) into (15), we obtain

$$
I_{l}=C_{l f} I_{f}+\sum_{r=1}^{n_{p}} C_{l r} I_{\mathrm{ent}, r} \quad \forall l=1, \ldots, n_{L}
$$

where $C_{l r}$ and $C_{l f}$ are constants, calculated by the BPZ parameters as

$$
\begin{array}{ll}
C_{l r}=\frac{Z_{l_{1} r}^{0}-Z_{l_{2} r}^{0}+\frac{y_{l}}{2} Z_{l_{1} r}^{0}}{z_{l}} & \forall r=1, \ldots, n_{b} \\
C_{l f}=C_{l f_{1}}+\left(C_{l f_{2}}-C_{l f_{1}}\right) x & \forall l=1, \ldots, n_{L} .
\end{array}
$$

Thus, (11) and (16) can be written in matrix form, as follows:

$$
H u=m
$$

where matrix $H$ consists of two sub-matrices $H_{V}$ and $H_{I}$ as follows:

$$
H=\left[\begin{array}{l}
H_{V} \\
H_{I}
\end{array}\right] .
$$

The dimensions of $H_{V}$ and $H_{I}$ are $n_{p} \times 2$ and $n_{L} \times 2$, respectively. These are constant matrices which are determined by BPZ parameters as

$$
H_{V_{i 1}}=Z_{i f_{1}}^{0} \quad i=1, \ldots, n_{p}
$$




$$
\begin{aligned}
H_{V_{i 2}} & =Z_{i f_{2}}^{0}-Z_{i f_{1}}^{0} & & i=1, \ldots, n_{p} \\
H_{I l 1} & =C_{l f_{1}} & & l=1, \ldots, n_{L} \\
H_{I l 2} & =C_{l f_{2}}-C_{l f_{1}} & & l=1, \ldots, n_{L} .
\end{aligned}
$$

In (19), vector $m$ consists of two sub-vectors $m_{V}, m_{I}$ as follows:

$$
m=\left[\begin{array}{l}
m_{V} \\
m_{I}
\end{array}\right] .
$$

The dimensions of $m_{V}$ and $m_{I}$ are $n_{p} \times 1$ and $n_{L} \times 1$, respectively. These are determined on the basis of the voltages and currents measured by PMUs and BPZ parameters, as

$$
\begin{array}{rlrl}
m_{V_{i}} & =V_{i}-\sum_{r=1}^{n_{p}} Z_{i r}^{0} I_{\mathrm{ent}, r} & i=1, \ldots, n_{p} \\
m_{I_{l}}=I_{l}-\sum_{r=1}^{n_{p}} C_{l r} I_{\mathrm{ent}, r} & l=1, \ldots, n_{L}
\end{array}
$$

and $u$ is the $2 \times 1$ unknown vector whose elements are as follows:

$$
u=\left[\begin{array}{l}
u_{1} \\
u_{2}
\end{array}\right]=\left[\begin{array}{c}
I_{f} \\
x I_{f}
\end{array}\right]
$$

If a fault occurs on one of the lines connected to a PMU bus, (15) and (16) are not valid for that line. Therefore, in (19), the related row is deleted and the dimension of matrix $H_{I}$ and vector $m_{I}$ will become $\left(n_{L}-1\right) \times 2$ and $\left(n_{L}-1\right) \times 1$, respectively.

In (19), matrix $H$ and vector $m$ are known and only vector $u$ is unknown. On the other hand, this equation is linear. Hence, $u$ can readily be estimated by the linear least squares method and afterwards $u_{1}, u_{2}$ are specified as

$$
\begin{aligned}
& u_{1}=\frac{1}{g}\left[\left(h_{2}^{*} \cdot h_{2}\right)\left(h_{1}^{*} \cdot m\right)-\left(h_{1}^{*} \cdot h_{2}\right)\left(h_{2}^{*} \cdot m\right)\right] \\
& u_{2}=\frac{1}{g}\left[\left(h_{1}^{*} \cdot h_{1}\right)\left(h_{2}^{*} \cdot m\right)-\left(h_{1} \cdot h_{2}^{*}\right)\left(h_{1}^{*} \cdot m\right)\right]
\end{aligned}
$$

where dot and asterisk denote inner product and complex conjugate, respectively. $h_{1}, h_{2}$ are first and second columns of matrix $H$, respectively. Moreover, $g$ is determined as follows:

$$
g=\left[\left(h_{1}^{*} \cdot h_{1}\right)\left(h_{2}^{*} \cdot h_{2}\right)-\left(h_{1}^{*} \cdot h_{2}\right)\left(h_{1} \cdot h_{2}^{*}\right)\right] .
$$

Therefore, estimation of the fault location, i.e., $x$ is acquired, as

$$
x=\frac{\left(h_{1}^{*} \cdot h_{1}\right)\left(h_{2}^{*} \cdot m\right)-\left(h_{1} \cdot h_{2}^{*}\right)\left(h_{1}^{*} \cdot m\right)}{\left(h_{2}^{*} \cdot h_{2}\right)\left(h_{1}^{*} \cdot m\right)-\left(h_{1}^{*} \cdot h_{2}\right)\left(h_{2}^{*} \cdot m\right)} .
$$

The purpose of this algorithm is identification of the faulted line in the faulted BPZ, i.e., the main unknown of this algorithm is the faulted line. For each line that belongs to the faulted BPZ, this algorithm first supposes that the fault has occurred on that line, and next estimates the fault location $x$ by (32). The faulted line is the line whose estimated $x$ is between 0 and 1 . If more than one line satisfy this condition, then, for each of these suspected lines the relative residual error of estimation is calculated as

$$
J(u)=\frac{\sqrt{\Delta u \cdot \Delta u^{*}}}{\sqrt{m \cdot m^{*}}}
$$

TABLE I

PMU Buses IN THE WSCC 9-Bus AND IEEE 118-Bus Test Systems

\begin{tabular}{c|c|c}
\hline $\begin{array}{c}\text { Test } \\
\text { system }\end{array}$ & PMU Buses & $\begin{array}{c}\text { No. of } \\
\text { PMUs }\end{array}$ \\
\hline $\begin{array}{c}\text { WSCC } \\
\text { 9-bus }\end{array}$ & $4,6,8$ & 3 \\
\hline IEEE & $\begin{array}{c}3,5,10,12,15,19,22,25,26,27,30,31,32, \\
69,42,43,46,49,51,54,56,59,61,65,66,\end{array}$ & 42 \\
118-bus & $\begin{array}{c}69,72,73,75,77,80,83,87,89,92,94,100, \\
103,105,110,111\end{array}$ & \\
\hline
\end{tabular}

TABLE II

LENGTHS OF TRANSMISSION LINES OF WSCC 9-BUS TEST SYSTEM

\begin{tabular}{|c||c|c|c|c|c|c|}
\hline Line & $4-5$ & $5-6$ & $6-7$ & $7-8$ & $8-9$ & $4-9$ \\
\hline Length $(\mathrm{km})$ & 122 & 225 & 133 & 95 & 213 & 112 \\
\hline
\end{tabular}

where

$$
\Delta u=H\left[\begin{array}{l}
u_{1} \\
u_{2}
\end{array}\right]-m
$$

The line, whose estimated $u$ best matches the measured values, is selected as the faulted line. In other words, the line which has the least relative residual error is selected as the faulted line.

In the above equations, the measurements have been assumed to have identical uncertainties. To deal with measurements with different uncertainties, it is sufficient that the linear weighted least squares method is used instead of linear least squares method [22], [23].

In order to compute the values of (29)-(34), it is sufficient to calculate a total number of seven inner products. In other words, the proposed algorithm provides a closedform and noniterative solution for the problem. Hence, this algorithm has a very low computation burden and so this method quickly identifies the faulted line. The flowchart of the proposed backup protection scheme is depicted in Fig. 3 .

\section{Simulation Results}

The WSCC 9-bus and IEEE 118-bus test systems are utilized to demonstrate the effectiveness of the adaptive wide area backup protection scheme. All simulations are executed by a $3.4 \mathrm{GHz}$ core i5 CPU with $4 \mathrm{~GB}$ of RAM.

The optimal PMU placement problem is modeled in GAMS optimization environment and the CPLEX solver is used to solve it. The result of optimal PMU placement is depicted in Table I. Next, BPZs are formed according to the network topology and PMU placement in MATLAB.

The WSCC 9-bus test system has six transmission lines [24]. The structure of this system along with its BPZs was shown in Fig. 1. In this figure, BPZs are specified by different contours. The lengths of transmission lines of this system are demonstrated in Table II. The IEEE 118-bus test system has 177 transmission lines [25], i.e., this system is similar to the largescale power systems. Thus, the performance of the protection scheme on the large-scale power systems can be validated by applying it to the IEEE 118-bus test system. This system are depicted in Fig. 4. 


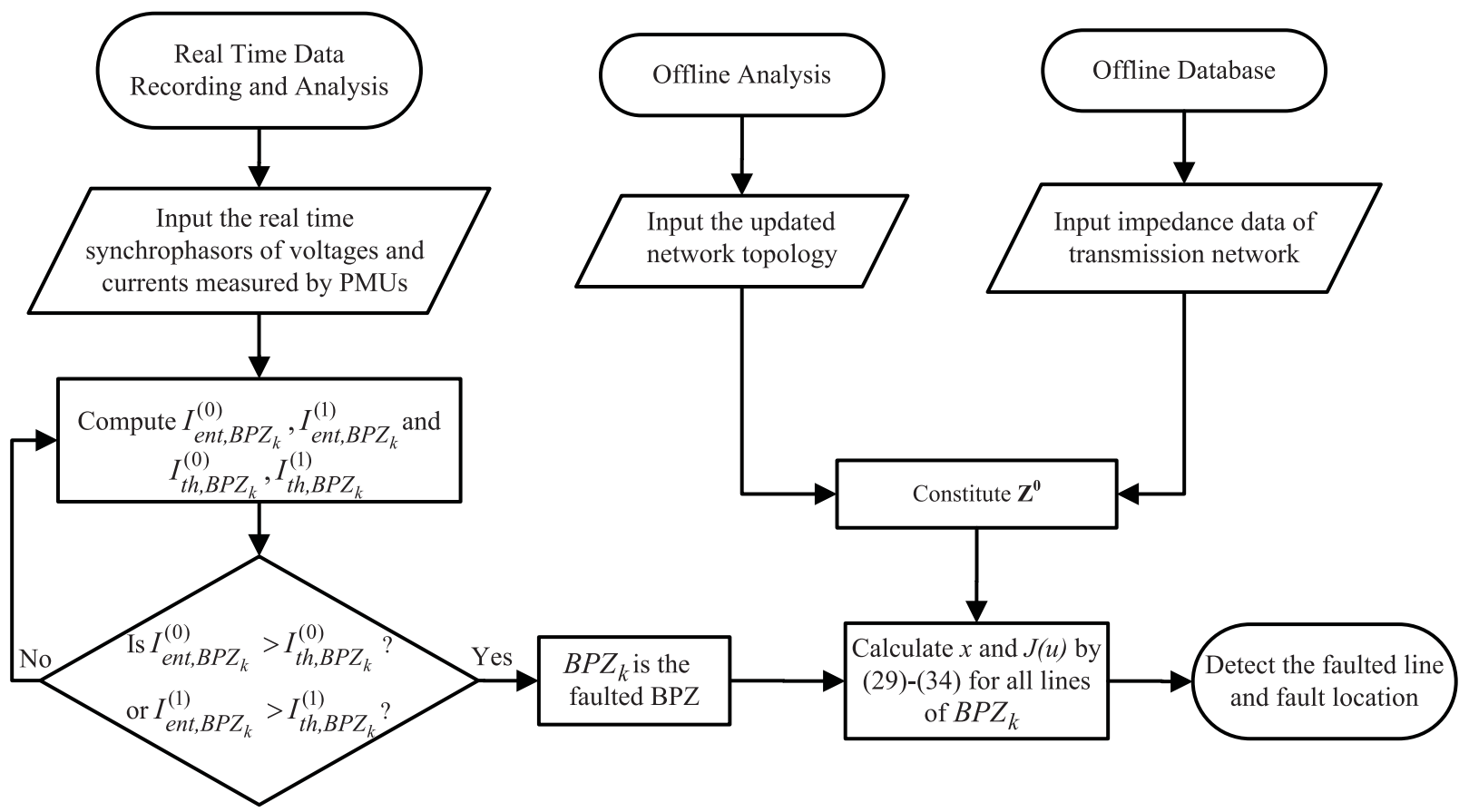

Fig. 3. Flowchart of the proposed wide-area backup protection scheme.

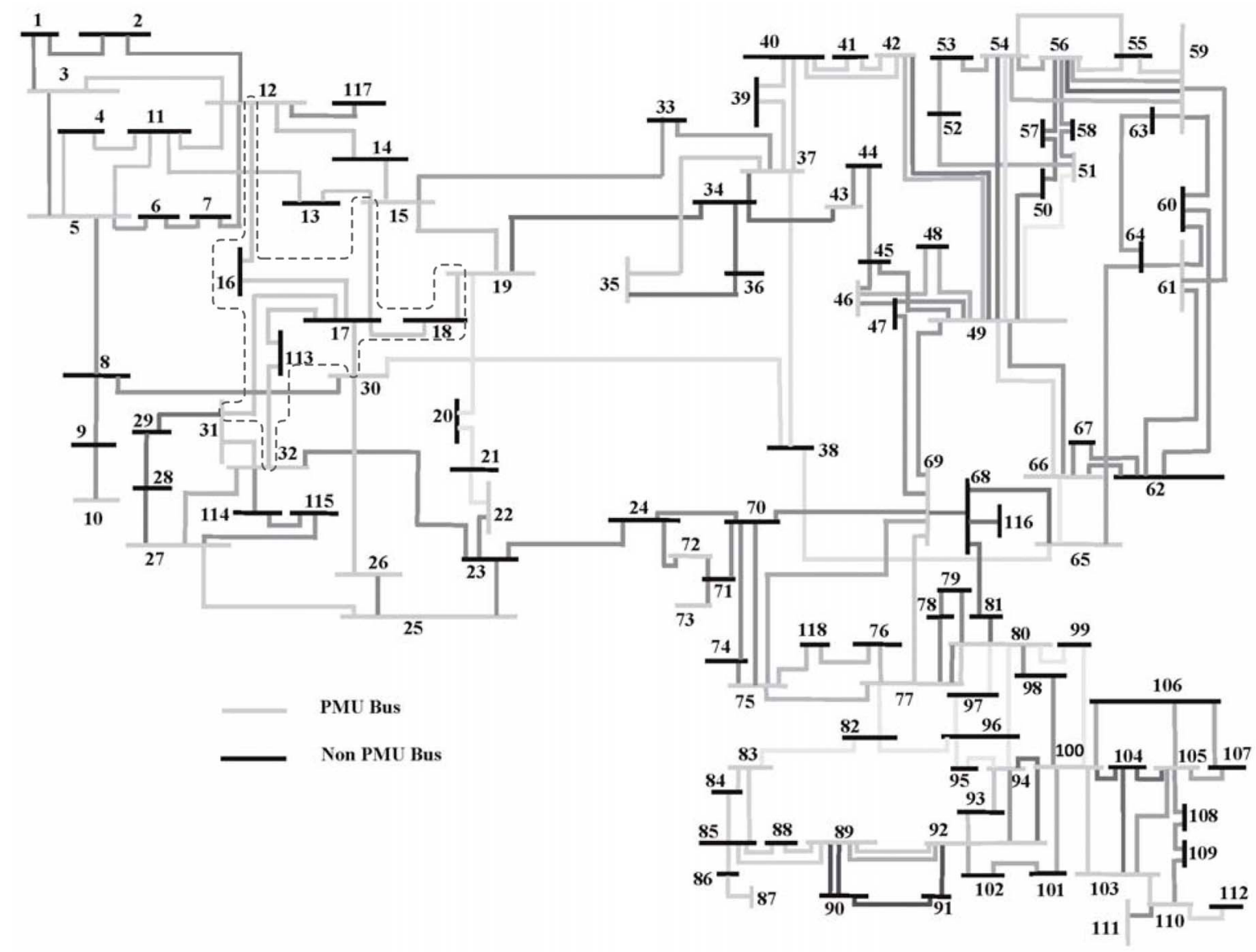

Fig. 4. IEEE 118-bus test system including 42 PMUs.

DIgSILENT Power Factory software is employed to simulate the electromagnetic transient of the fault. PMUs extract the fundamental component of input signals in four stages which are modeled in MATLAB environment. First the input signals are passed through a second-order Butterworth anti-aliasing filter with a cut-off frequency of $350 \mathrm{~Hz}$, which removes high 
TABLE III

LENGTHS OF TRANSMISSION LINES OF BPZ1 OF IEEE 118-BUS TEST SYSTEM

\begin{tabular}{|c||c|c|c|c|c|c|c|c|}
\hline Line & $12-16$ & $15-17$ & $16-17$ & $17-18$ & $17-31$ & $17-113$ & $18-19$ & $32-113$ \\
\hline Length (km) & 45 & 24 & 97 & 28 & 84 & 17 & 27 & 140 \\
\hline
\end{tabular}

TABLE IV

Thresholds OF WSCC 9-Bus AND IEEE 118-Bus Test Systems

\begin{tabular}{c|c|c|c}
\hline \multirow{2}{*}{ Test system } & BPZ Number & $I_{t h, B P Z}^{(0)}(p . u)$. & $I_{t h, B P Z}^{(1)}(p . u)$. \\
\hline \multirow{3}{*}{ WSCC 9-bus } & BPZ1 & 0.218 & 2.181 \\
\cline { 2 - 4 } & BPZ2 & 0.200 & 1.550 \\
\cline { 2 - 4 } & BPZ3 & 0.200 & 1.684 \\
\hline IEEE 118-bus & BPZ1 & 0.551 & 2.203 \\
\hline
\end{tabular}

frequency harmonics of input signals. In the second stage, the output signal of Butterworth filter is sampled at $4800 \mathrm{~Hz}$ which is equal to 96 samples per cycle. In the third stage, a digital mimic filter is used to eliminate the decaying dc offset [26]. In the last stage, the fundamental components of input signals are obtained by using full-cycle Fourier transform.

MATLAB software is used to construct the bus impedance matrix $Z^{0}$ for all BPZs. On the basis of network topology, bus impedance matrix is updated and matrix $H$ and vector $m$ are calculated as discussed in Section IV.

Faults on the WSCC 9-bus test system are thoroughly studied and in order to save space only the results of faults in BPZ1 of IEEE 118-bus test system will be thoroughly reported. BPZ1 consists of 10 buses and 8 transmission lines. The lengths of transmission lines of this BPZ are demonstrated in Table III. This BPZ are specified by a contour in Fig. 4. This BPZ has four non-PMU buses 16, 17, 18 and 113 which are surrounded by six PMU buses 12, 15, 19, 30, 31, and 32. The proposed adaptive wide area backup protection scheme consists of two stages. First it detects the faulted zone and next determines the faulted line and fault location. In the following, these stages are investigated separately.

\section{A. Faulted Zone Identification}

The threshold values of zero- and positive-sequence currents entering the BPZs of the two test systems are demonstrated in Table IV. For all BPZs, values of $d_{0}$ and $d_{1}$ are considered to be 0.2 and 2, respectively. For all BPZs, the value of $I_{\min , \mathrm{BPZ}_{k}}$ is set to 0.2 p.u. Various balanced and unbalanced faults are applied to different distances of lines. In all studies, fault resistance values are assumed to be $100 \Omega$ for AG and BCG types and $1 \Omega$ for BC and ABCG types, unless specified otherwise. Tables V and VI show the values of $I_{\mathrm{ent}, \mathrm{BPZ}}^{(s)}$ for various BPZs. In these tables, values which exceed the corresponding thresholds are represented in the bold font. It can be seen that based on (7), the faulted zone has been identified for all fault cases correctly.

In order to examine the efficiency of the faulted zone identification algorithm in very short and long transmission lines, it is applied to the IEEE 118-bus test system. For all fault types (AG, BC, BCG, ABCG) at various fault locations $(5 \%, 50 \%, 95 \%)$ on every transmission line of this system,
TABLE V

Fault Zone IDENTIFICATION OF WSCC 9-Bus Test System

\begin{tabular}{|c|c|c|c|c|c|c|}
\hline $\begin{array}{c}\text { Faulted } \\
\text { Line }\end{array}$ & $\begin{array}{l}\text { Fault } \\
\text { Type }\end{array}$ & \begin{tabular}{|c|} 
Fault \\
Distance \\
(p.u.)
\end{tabular} & $k$ & $\begin{array}{l}I_{\text {ent }, B P Z_{k}}^{(0)} \\
\quad(p . u .)\end{array}$ & $\begin{array}{l}I_{\text {ent }, B P Z_{k}}^{(1)} \\
\quad(\text { p.u. })\end{array}$ & $\begin{array}{l}\text { Identified } \\
\text { BPZ }\end{array}$ \\
\hline \multirow{3}{*}{$4-5$} & \multirow{3}{*}{ AG } & \multirow{3}{*}{0.1} & 1 & 0.047 & 1.177 & \multirow{3}{*}{ BPZ2 } \\
\hline & & & 2 & 1.327 & 2.015 & \\
\hline & & & 3 & 0.012 & 0.953 & \\
\hline \multirow{3}{*}{$5-6$} & \multirow{3}{*}{ BC } & \multirow{3}{*}{0.3} & 1 & 0.000 & 0.958 & \multirow{3}{*}{ BPZ2 } \\
\hline & & & 2 & 0.000 & 3.354 & \\
\hline & & & 3 & 0.000 & 0.760 & \\
\hline \multirow{3}{*}{$6-7$} & \multirow{3}{*}{ BCG } & \multirow{3}{*}{0.5} & 1 & 0.024 & 1.152 & \multirow{3}{*}{ BPZ3 } \\
\hline & & & 2 & 0.038 & 0.828 & \\
\hline & & & 3 & 1.200 & 3.196 & \\
\hline \multirow{3}{*}{$7-8$} & \multirow{3}{*}{$\mathrm{ABCG}$} & \multirow{3}{*}{0.7} & 1 & 0.000 & 0.521 & \multirow{3}{*}{ BPZ3 } \\
\hline & & & 2 & 0.000 & 0.458 & \\
\hline & & & 3 & 0.001 & 7.627 & \\
\hline \multirow{3}{*}{$8-9$} & \multirow{3}{*}{ AG } & \multirow{3}{*}{0.9} & 1 & 1.145 & 2.112 & \multirow{3}{*}{ BPZ1 } \\
\hline & & & 2 & 0.033 & 0.861 & \\
\hline & & & 3 & 0.020 & 0.942 & \\
\hline \multirow{3}{*}{$4-9$} & \multirow{3}{*}{$\mathrm{BC}$} & \multirow{3}{*}{0.1} & 1 & 0.000 & 4.530 & \multirow{3}{*}{ BPZ1 } \\
\hline & & & 2 & 0.000 & 0.555 & \\
\hline & & & 3 & 0.001 & 0.760 & \\
\hline
\end{tabular}

TABLE VI

Fault Zone Identification of IEEE 118-Bus Test System

\begin{tabular}{c|c|c|c|c|c|c}
\hline $\begin{array}{c}\text { Faulted } \\
\text { Line }\end{array}$ & $\begin{array}{c}\text { Fault } \\
\text { Type }\end{array}$ & $\begin{array}{c}\text { Fault } \\
\text { Distance } \\
\text { (p.u.) }\end{array}$ & $k$ & $\begin{array}{c}I_{\text {ent }, B P Z_{k}}^{(0)} \\
(p . u .)\end{array}$ & $\begin{array}{c}I_{\text {ent }, B P Z_{k}}^{(1)}(p . u .) \\
\text { Identified } \\
\text { BPZ }\end{array}$ \\
\hline $12-16$ & AG & 0.1 & 1 & $\mathbf{0 . 6 1 8}$ & 1.745 & BPZ1 \\
\hline $15-17$ & BC & 0.3 & 1 & 0.000 & $\mathbf{9 . 7 8 7}$ & BPZ1 \\
\hline $16-17$ & BCG & 0.5 & 1 & $\mathbf{0 . 5 7 2}$ & $\mathbf{2 . 2 9 2}$ & BPZ1 \\
\hline $17-18$ & ABCG & 0.7 & 1 & 0.000 & $\mathbf{1 5 . 4 1 5}$ & BPZ1 \\
\hline $17-31$ & AG & 0.9 & 1 & $\mathbf{0 . 5 9 5}$ & 1.727 & BPZ1 \\
\hline $17-113$ & BC & 0.1 & 1 & 0.000 & $\mathbf{1 1 . 7 2 3}$ & BPZ1 \\
\hline $18-19$ & BCG & 0.3 & 1 & $\mathbf{0 . 5 8 3}$ & $\mathbf{2 . 2 8 7}$ & BPZ1 \\
\hline $32-113$ & ABCG & 0.5 & 1 & 0.000 & $\mathbf{1 0 . 3 0 9}$ & BPZ1 \\
\hline
\end{tabular}

the proposed algorithm correctly identifies the faulted BPZ. This is due to the fact that the thresholds of each BPZ have been defined in adaptive manner and thus the efficiency of this algorithm is independent of transmission line length.

\section{B. Faulted Line Identification}

Table VII reflects the results of faulted line identification and fault location estimation for various fault types occurred at different points of WSCC 9-bus test system. For all fault types occurred on the lines of this system, the faulted line identification algorithm accurately identifies the faulted line and fault location.

In order to measure the precision of fault location estimation, the fault location estimation error is defined as

$$
\text { FL Error }(\%)=\frac{\mid \text { Estimated } F L-\text { Actual } F L \mid}{\text { Line Length }} \times 100 \text {. }
$$

The single phase to ground fault is the most common fault in transmission lines. So the efficacy of this scheme for AG fault type is investigated in Table VIII. This table shows the results of faulted line identification and fault location estimation for different fault resistance values and various points of WSCC 9-bus test system. 
TABLE VII

INFLUENCE OF FAULT TyPE ON FAULTED LINE IDENTIFICATION ALGORITHM FOR WSCC 9-BUS TEST SySTEM

\begin{tabular}{|c|c|c|c|c|c|c|}
\hline \multirow{3}{*}{$\begin{array}{l}\text { Faulted } \\
\text { Line }\end{array}$} & \multirow{3}{*}{\begin{tabular}{|c|} 
Fault \\
Distance \\
(p.u.)
\end{tabular}} & \multicolumn{4}{|c|}{ FL estimation error (\%) } & \multirow{3}{*}{$\begin{array}{l}\text { Identified } \\
\text { Faulted } \\
\text { Line }\end{array}$} \\
\hline & & \multicolumn{4}{|c|}{ Fault Type } & \\
\hline & & $\mathrm{AG}$ & $\mathrm{BC}$ & BCG & ABCG & \\
\hline \multirow{5}{*}{$4-5$} & 0.1 & 0.266 & 1.349 & 0.155 & 2.400 & $4-5$ \\
\hline & 0.3 & 0.270 & 1.020 & 0.009 & 1.925 & $4-5$ \\
\hline & 0.5 & 0.251 & 0.739 & 0.136 & 1.530 & $4-5$ \\
\hline & 0.7 & 0.217 & 0.512 & 0.150 & 1.208 & $4-5$ \\
\hline & 0.9 & 0.157 & 0.350 & 0.095 & 0.967 & $4-5$ \\
\hline \multirow{5}{*}{$5-6$} & 0.1 & 0.073 & 0.183 & 0.078 & 0.540 & $5-6$ \\
\hline & 0.3 & 0.147 & 0.058 & 0.144 & 0.397 & $5-6$ \\
\hline & 0.5 & 0.075 & 0.206 & 0.139 & 0.055 & $5-6$ \\
\hline & 0.7 & 0.016 & 0.543 & 0.049 & 0.407 & $5-6$ \\
\hline & 0.9 & 0.045 & 0.911 & 0.071 & 0.983 & $5-6$ \\
\hline \multirow{5}{*}{$6-7$} & 0.1 & 0.276 & 1.120 & 0.177 & 2.192 & $6-7$ \\
\hline & 0.3 & 0.178 & 0.733 & 0.115 & 1.512 & $6-7$ \\
\hline & 0.5 & 0.164 & 0.375 & 0.093 & 0.990 & $6-7$ \\
\hline & 0.7 & 0.139 & 0.103 & 0.094 & 0.429 & $6-7$ \\
\hline & 0.9 & 0.065 & 0.113 & 0.047 & 0.053 & 6-7 \\
\hline \multirow{5}{*}{$7-8$} & 0.1 & 0.143 & 0.342 & 0.062 & 0.400 & $7-8$ \\
\hline & 0.3 & 0.222 & 0.562 & 0.096 & 0.853 & $7-8$ \\
\hline & 0.5 & 0.338 & 0.866 & 0.058 & 1.378 & $7-8$ \\
\hline & 0.7 & 0.395 & 1.204 & 0.046 & 2.042 & $7-8$ \\
\hline & 0.9 & 0.556 & 1.602 & 0.061 & 2.736 & $7-8$ \\
\hline \multirow{5}{*}{$8-9$} & 0.1 & 0.776 & 0.189 & 0.654 & 1.404 & $8-9$ \\
\hline & 0.3 & 0.707 & 0.096 & 0.660 & 0.701 & $8-9$ \\
\hline & 0.5 & 0.624 & 0.296 & 0.637 & 0.111 & $8-9$ \\
\hline & 0.7 & 0.464 & 0.379 & 0.491 & 0.327 & $8-9$ \\
\hline & 0.9 & 0.184 & 0.300 & 0.225 & 0.569 & $8-9$ \\
\hline \multirow{5}{*}{$4-9$} & 0.1 & 1.166 & 0.624 & 0.573 & 2.768 & $4-9$ \\
\hline & 0.3 & 1.013 & 0.384 & 0.644 & 2.193 & $4-9$ \\
\hline & 0.5 & 0.825 & 0.231 & 0.575 & 1.716 & $4-9$ \\
\hline & 0.7 & 0.629 & 0.182 & 0.376 & 1.343 & $4-9$ \\
\hline & 0.9 & 0.341 & 0.232 & 0.071 & 1.036 & $4-9$ \\
\hline
\end{tabular}

In order to examine the efficiency of the faulted line identification algorithm in large power systems, it is applied to the IEEE 118-bus test system. The obtained results for BPZ1 of this system are gathered in Tables IX and X.

In order to investigate the effect of transmission line length on the efficiency of the faulted line identification algorithm, all transmission lines of IEEE 118-bus test system are classified into six groups according to the transmission line length. Afterwards, all fault types (AG, BC, BCG, ABCG) are simulated at various fault locations $(5 \%, 50 \%, 95 \%)$ on every transmission line of each group. For all simulations, this algorithm accurately identifies the faulted line and fault location. The obtained results are summarized in Table XI. This table provides the maximum fault location estimation error for each group of transmission lines; this value of fault location estimation error is acceptable, since the proposed algorithm must quickly identify the faulted line, i.e., it must have low computational burden.

According to Table XI, if the distance between the fault location on the faulted line and the nearest bus to the fault is less than the maximum fault location estimation error of the faulted line, it is possible that the proposed algorithm cannot determine the faulted line and fault location. This problem is negligible, since the maximum fault location estimation error is very small.
TABLE VIII

InFLUENCE OF FAULt RESISTANCE ON FAULTEd Line IdENTIFICATION AlgORITHM FOR WSCC 9-BUS TEST SYSTEM

\begin{tabular}{|c|c|c|c|c|c|c|}
\hline \multirow{3}{*}{$\begin{array}{l}\text { Faulted } \\
\text { Line }\end{array}$} & \multirow{3}{*}{\begin{tabular}{|c|} 
Fault \\
Distance \\
(p.u.)
\end{tabular}} & \multirow{2}{*}{\multicolumn{4}{|c|}{$\begin{array}{c}\text { FL estimation error }(\%) \\
\text { Fault Resistance }(\Omega)\end{array}$}} & \multirow{3}{*}{$\begin{array}{l}\text { Identified } \\
\text { Faulted } \\
\text { Line }\end{array}$} \\
\hline & & & & & & \\
\hline & & 1 & 10 & 50 & 100 & \\
\hline \multirow{5}{*}{$4-5$} & 0.1 & 0.659 & 0.549 & 0.135 & 0.266 & $4-5$ \\
\hline & 0.3 & 0.514 & 0.400 & 0.141 & 0.270 & $4-5$ \\
\hline & 0.5 & 0.368 & 0.310 & 0.112 & 0.251 & $4-5$ \\
\hline & 0.7 & 0.070 & 0.010 & 0.147 & 0.217 & $4-5$ \\
\hline & 0.9 & 0.064 & 0.062 & 0.101 & 0.157 & $4-5$ \\
\hline \multirow{5}{*}{$5-6$} & 0.1 & 0.125 & 0.130 & 0.095 & 0.073 & $5-6$ \\
\hline & 0.3 & 0.193 & 0.190 & 0.179 & 0.147 & $5-6$ \\
\hline & 0.5 & 0.098 & 0.087 & 0.115 & 0.075 & $5-6$ \\
\hline & 0.7 & 0.224 & 0.196 & 0.009 & 0.016 & $5-6$ \\
\hline & 0.9 & 0.506 & 0.461 & 0.060 & 0.045 & $5-6$ \\
\hline \multirow{5}{*}{ 6-7 } & 0.1 & 0.548 & 0.470 & 0.163 & 0.276 & $6-7$ \\
\hline & 0.3 & 0.266 & 0.242 & 0.117 & 0.178 & 6-7 \\
\hline & 0.5 & 0.104 & 0.102 & 0.117 & 0.164 & 6-7 \\
\hline & 0.7 & 0.051 & 0.044 & 0.118 & 0.139 & 6-7 \\
\hline & 0.9 & 0.050 & 0.064 & 0.066 & 0.065 & $6-7$ \\
\hline \multirow{5}{*}{$7-8$} & 0.1 & 0.123 & 0.105 & 0.101 & 0.143 & $7-8$ \\
\hline & 0.3 & 0.089 & 0.092 & 0.170 & 0.222 & $7-8$ \\
\hline & 0.5 & 0.111 & 0.135 & 0.240 & 0.338 & $7-8$ \\
\hline & 0.7 & 0.385 & 0.493 & 0.223 & 0.395 & $7-8$ \\
\hline & 0.9 & 0.754 & 0.696 & 0.328 & 0.556 & $7-8$ \\
\hline \multirow{5}{*}{$8-9$} & 0.1 & 0.290 & 0.310 & 0.722 & 0.776 & $8-9$ \\
\hline & 0.3 & 0.468 & 0.478 & 0.680 & 0.707 & 8-9 \\
\hline & 0.5 & 0.644 & 0.618 & 0.637 & 0.624 & $8-9$ \\
\hline & 0.7 & 0.565 & 0.552 & 0.508 & 0.464 & 8-9 \\
\hline & 0.9 & 0.295 & 0.293 & 0.224 & 0.184 & $8-9$ \\
\hline \multirow{5}{*}{$4-9$} & 0.1 & 0.379 & 0.310 & 0.997 & 1.166 & $4-9$ \\
\hline & 0.3 & 0.185 & 0.201 & 0.818 & 1.013 & $4-9$ \\
\hline & 0.5 & 0.434 & 0.357 & 0.670 & 0.825 & $4-9$ \\
\hline & 0.7 & 0.333 & 0.307 & 0.531 & 0.629 & $4-9$ \\
\hline & 0.9 & 0.006 & 0.005 & 0.231 & 0.341 & $4-9$ \\
\hline
\end{tabular}

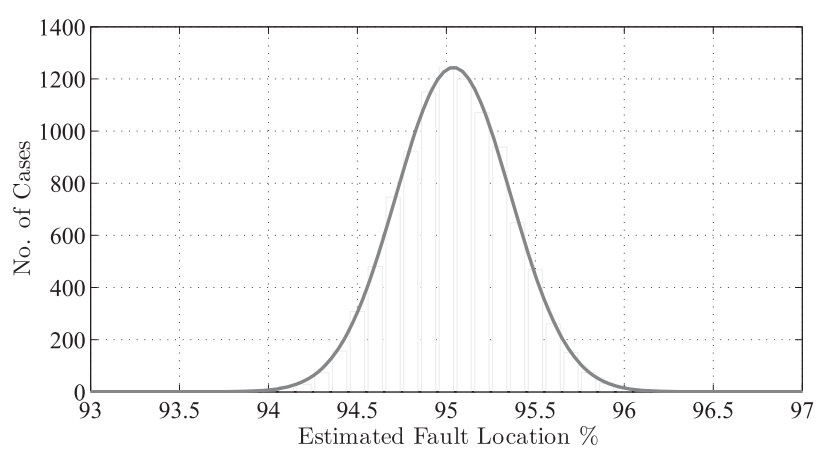

Fig. 5. Influence of measurement errors on faulted line identification algorithm for single phase to ground faults at $95 \%$ of the lines 16 and 17 .

In order to verify the robustness of the faulted line identification algorithm against the measurement errors, $\pm 5 \%$ p.u. amplitude error and $\pm 1^{\circ}$ phase error are considered for measurements. It is assumed that these errors have normal distribution with zero mean. The acquired results for a total of 10000 single phase to ground fault cases at $95 \%$ of the lines 16-17 is shown in Fig. 5. Although this line is not equipped with any PMUs, the proposed algorithm accurately identifies the faulted line and fault location. As shown, the estimated fault locations demonstrate normal distribution with a mean of $95.04 \%$ and standard deviation $0.4551 \%$. 
TABLE IX

INFLUENCE OF FAULT TYPE ON FAULTED LINE IDENTIFICATION ALGORITHM FOR IEEE 118-Bus TEST System

\begin{tabular}{|c|c|c|c|c|c|c|}
\hline \multirow{3}{*}{$\begin{array}{c}\text { Faulted } \\
\text { Line }\end{array}$} & \multirow{3}{*}{\begin{tabular}{|c|} 
Fault \\
Distance \\
(p.u.)
\end{tabular}} & \multicolumn{4}{|c|}{ FL estimation error $(\%)$} & \multirow{3}{*}{$\begin{array}{l}\text { Identified } \\
\text { Faulted } \\
\text { Line }\end{array}$} \\
\hline & & \multicolumn{4}{|c|}{ Fault Type } & \\
\hline & & AG & $\mathrm{BC}$ & BCG & ABCG & \\
\hline \multirow{5}{*}{$12-16$} & 0.1 & 0.764 & 0.380 & 0.492 & 0.679 & $12-16$ \\
\hline & 0.3 & 0.751 & 0.464 & 0.411 & 0.935 & $12-16$ \\
\hline & 0.5 & 0.819 & 0.518 & 0.383 & 1.158 & $12-16$ \\
\hline & 0.7 & 0.873 & 0.559 & 0.310 & 1.329 & $12-16$ \\
\hline & 0.9 & 0.899 & 0.592 & 0.241 & 1.454 & $12-16$ \\
\hline \multirow{5}{*}{$15-17$} & 0.1 & 1.795 & 1.558 & 1.664 & 0.855 & $15-17$ \\
\hline & 0.3 & 1.809 & 1.647 & 1.777 & 0.778 & $15-17$ \\
\hline & 0.5 & 1.790 & 1.736 & 1.914 & 0.633 & $15-17$ \\
\hline & 0.7 & 1.860 & 1.824 & 1.964 & 0.437 & $15-17$ \\
\hline & 0.9 & 1.988 & 1.913 & 1.968 & 0.201 & $15-17$ \\
\hline \multirow{5}{*}{$16-17$} & 0.1 & 0.419 & 0.286 & 0.070 & 0.734 & 16-17 \\
\hline & 0.3 & 0.417 & 0.311 & 0.003 & 0.729 & $16-17$ \\
\hline & 0.5 & 0.435 & 0.346 & 0.081 & 0.628 & $16-17$ \\
\hline & 0.7 & 0.446 & 0.384 & 0.144 & 0.445 & $16-17$ \\
\hline & 0.9 & 0.438 & 0.419 & 0.218 & 0.167 & $16-17$ \\
\hline \multirow{5}{*}{$17-18$} & 0.1 & 1.557 & 1.583 & 1.692 & 0.156 & $17-18$ \\
\hline & 0.3 & 1.488 & 1.543 & 1.716 & 0.434 & $17-18$ \\
\hline & 0.5 & 1.457 & 1.501 & 1.685 & 0.701 & $17-18$ \\
\hline & 0.7 & 1.461 & 1.455 & 1.618 & 0.940 & $17-18$ \\
\hline & 0.9 & 1.454 & 1.411 & 1.570 & 1.110 & $17-18$ \\
\hline \multirow{5}{*}{$17-31$} & 0.1 & 0.433 & 0.424 & 0.321 & 0.096 & $17-31$ \\
\hline & 0.3 & 0.483 & 0.386 & 0.190 & 0.367 & $17-31$ \\
\hline & 0.5 & 0.500 & 0.335 & 0.061 & 0.556 & $17-31$ \\
\hline & 0.7 & 0.439 & 0.279 & 0.042 & 0.651 & $17-31$ \\
\hline & 0.9 & 0.336 & 0.222 & 0.122 & 0.651 & $17-31$ \\
\hline \multirow{5}{*}{$17-113$} & 0.1 & 2.369 & 2.214 & 2.014 & 0.202 & $17-113$ \\
\hline & 0.3 & 2.234 & 2.190 & 1.958 & 0.381 & $17-113$ \\
\hline & 0.5 & 2.139 & 2.177 & 2.001 & 0.582 & $17-113$ \\
\hline & 0.7 & 2.098 & 2.158 & 2.038 & 0.857 & $17-113$ \\
\hline & 0.9 & 2.090 & 2.135 & 2.059 & 1.156 & $17-113$ \\
\hline \multirow{5}{*}{$18-19$} & 0.1 & 1.458 & 1.410 & 1.587 & 1.258 & 18-19 \\
\hline & 0.3 & 1.430 & 1.372 & 1.575 & 1.329 & $18-19$ \\
\hline & 0.5 & 1.391 & 1.331 & 1.567 & 1.346 & $18-19$ \\
\hline & 0.7 & 1.338 & 1.293 & 1.544 & 1.299 & $18-19$ \\
\hline & 0.9 & 1.248 & 1.255 & 1.545 & 1.188 & $18-19$ \\
\hline \multirow{5}{*}{$32-113$} & 0.1 & 0.248 & 0.153 & 0.304 & 0.539 & $32-113$ \\
\hline & 0.3 & 0.057 & 0.191 & 0.226 & 0.641 & $32-113$ \\
\hline & 0.5 & 0.275 & 0.234 & 0.163 & 0.625 & $32-113$ \\
\hline & 0.7 & 0.314 & 0.276 & 0.233 & 0.515 & $32-113$ \\
\hline & 0.9 & 0.324 & 0.306 & 0.292 & 0.323 & $32-113$ \\
\hline
\end{tabular}

All results of Tables $\mathrm{V}-\mathrm{XI}$ indicate that the performance of the method is not affected by high-impedance faults. Moreover, for different fault types, the faulted BPZ and faulted line can be identified correctly. Furthermore, this scheme determines the fault location precisely. For all cases, the fault location estimation error is very small. The reason is that the conventional protection schemes ignore the fault resistance (assume that the fault resistance is equal to zero), but this scheme models the fault as a current source. Therefore, in comparison with conventional schemes, this scheme is not sensitive to the fault type and resistance.

In order to investigate the speed of the proposed wide area backup protection scheme, the execution time (ET) of this scheme is computed. The ET of this scheme is equal to the ET of the faulted zone identification algorithm plus the ET of the faulted line identification algorithm. For all fault cases, the
TABLE $X$

INFLUENCE OF FAULT RESISTANCE ON FAULTED LINE IDENTIFICATION ALGORITHM FOR IEEE 118 -BUS TEST SYSTEM

\begin{tabular}{|c|c|c|c|c|c|c|}
\hline \multirow{3}{*}{$\begin{array}{l}\text { Faulted } \\
\text { Line }\end{array}$} & \multirow{3}{*}{$\left|\begin{array}{c}\text { Fault } \\
\text { Distance } \\
\text { (p.u.) }\end{array}\right|$} & \multirow{2}{*}{\multicolumn{4}{|c|}{$\begin{array}{c}\text { FL estimation error }(\%) \\
\text { Fault Resistance }(\Omega)\end{array}$}} & \multirow{3}{*}{$\begin{array}{c}\text { Identified } \\
\text { Faulted } \\
\text { Line }\end{array}$} \\
\hline & & & & & & \\
\hline & & 1 & 10 & 50 & 100 & \\
\hline \multirow{5}{*}{$12-16$} & 0.1 & 0.690 & 0.875 & 0.835 & 0.764 & $12-16$ \\
\hline & 0.3 & 0.557 & 0.777 & 0.890 & 0.751 & $12-16$ \\
\hline & 0.5 & 0.499 & 0.699 & 0.941 & 0.819 & $12-16$ \\
\hline & 0.7 & 0.476 & 0.648 & 0.943 & 0.873 & $12-16$ \\
\hline & 0.9 & 0.449 & 0.606 & 0.924 & 0.899 & $12-16$ \\
\hline \multirow{5}{*}{$15-17$} & 0.1 & 2.476 & 2.199 & 1.812 & 1.795 & 15-17 \\
\hline & 0.3 & 2.443 & 2.175 & 1.770 & 1.809 & $15-17$ \\
\hline & 0.5 & 2.373 & 2.047 & 1.671 & 1.790 & $15-17$ \\
\hline & 0.7 & 2.108 & 1.750 & 1.712 & 1.860 & $15-17$ \\
\hline & 0.9 & 1.354 & 1.527 & 1.842 & 1.988 & 15-17 \\
\hline \multirow{5}{*}{$16-17$} & 0.1 & 0.219 & 0.277 & 0.413 & 0.419 & $16-17$ \\
\hline & 0.3 & 0.229 & 0.278 & 0.401 & 0.417 & $16-17$ \\
\hline & 0.5 & 0.280 & 0.317 & 0.419 & 0.435 & $16-17$ \\
\hline & 0.7 & 0.284 & 0.325 & 0.438 & 0.446 & 16-17 \\
\hline & 0.9 & 0.301 & 0.374 & 0.443 & 0.438 & $16-17$ \\
\hline \multirow{5}{*}{$17-18$} & 0.1 & 1.144 & 1.245 & 1.460 & 1.557 & $17-18$ \\
\hline & 0.3 & 1.783 & 1.523 & 1.392 & 1.488 & $17-18$ \\
\hline & 0.5 & 1.985 & 1.782 & 1.443 & 1.457 & $17-18$ \\
\hline & 0.7 & 2.067 & 1.892 & 1.520 & 1.461 & $17-18$ \\
\hline & 0.9 & 2.130 & 1.948 & 1.546 & 1.454 & $17-18$ \\
\hline \multirow{5}{*}{$17-31$} & 0.1 & 0.291 & 0.324 & 0.382 & 0.433 & $17-31$ \\
\hline & 0.3 & 0.403 & 0.440 & 0.484 & 0.483 & $17-31$ \\
\hline & 0.5 & 0.357 & 0.425 & 0.522 & 0.500 & $17-31$ \\
\hline & 0.7 & 0.275 & 0.353 & 0.479 & 0.439 & $17-31$ \\
\hline & 0.9 & 0.227 & 0.291 & 0.386 & 0.336 & $17-31$ \\
\hline \multirow{5}{*}{$17-113$} & 0.1 & 1.602 & 1.891 & 2.202 & 2.369 & $17-113$ \\
\hline & 0.3 & 1.493 & 1.711 & 1.980 & 2.234 & $17-113$ \\
\hline & 0.5 & 1.345 & 1.510 & 1.851 & 2.139 & $17-113$ \\
\hline & 0.7 & 1.388 & 1.496 & 1.827 & 2.098 & $17-113$ \\
\hline & 0.9 & 1.502 & 1.548 & 1.844 & 2.090 & $17-113$ \\
\hline \multirow{5}{*}{$18-19$} & 0.1 & 2.234 & 2.023 & 1.566 & 1.458 & $18-19$ \\
\hline & 0.3 & 2.301 & 2.068 & 1.557 & 1.430 & 18-19 \\
\hline & 0.5 & 2.342 & 2.077 & 1.525 & 1.391 & $18-19$ \\
\hline & 0.7 & 2.374 & 2.079 & 1.485 & 1.338 & $18-19$ \\
\hline & 0.9 & 2.321 & 1.968 & 1.384 & 1.248 & $18-19$ \\
\hline \multirow{5}{*}{$32-113$} & 0.1 & 0.041 & 0.019 & 0.119 & 0.248 & $32-113$ \\
\hline & 0.3 & 0.264 & 0.273 & 0.195 & 0.057 & $32-113$ \\
\hline & 0.5 & 0.344 & 0.368 & 0.362 & 0.275 & $32-113$ \\
\hline & 0.7 & 0.317 & 0.329 & 0.334 & 0.314 & $32-113$ \\
\hline & 0.9 & 0.290 & 0.288 & 0.303 & 0.324 & $32-113$ \\
\hline
\end{tabular}

TABLE XI

INFLUENCE OF TRANSMISSION LINE LENGTH ON FAULTED LINE IDENTIFICATION ALGORITHM FOR IEEE 118-Bus TEST SYSTEM

\begin{tabular}{|c||c|c|c|c|c|c|}
\hline $\begin{array}{c}\text { Transmission Line } \\
\text { Length (km) }\end{array}$ & $0-100$ & $100-150$ & $150-200$ & $200-250$ & $250-300$ & $300-450$ \\
\hline $\begin{array}{c}\text { Maximum Fault } \\
\text { Location Estimation } \\
\text { Error (\%) }\end{array}$ & 2.865 & 2.781 & 2.193 & 1.627 & 1.141 & 0.782 \\
\hline
\end{tabular}

proposed method is executed quickly. The ET of the proposed adaptive wide area backup protection method is estimated to be $3 \mathrm{~ms}$ for all fault conditions. On the other hand, it has been assumed that the proposed backup protection scheme is implemented in the local control center. The communication delay of WAMS is less than $200 \mathrm{~ms}$ in either direction [14], [27]. 
Therefore, even if the primary protection fails, the backup protection scheme isolates the faulted line less than $403 \mathrm{~ms}$ after the fault occurrs. According to the results, both stages of the proposed backup protection scheme have a successful performance. This scheme not only protects transmission lines but also precisely determines the fault location. Therefore, similar to the conventional protective relays the fault location function can be included in the proposed scheme.

\section{CONCLUSION}

An adaptive wide area backup protection scheme for transmission lines has been proposed. It utilizes wide area data of WAMS and bus impedance matrix of BPZs instead of local data and impedance of the faulted line. This protection scheme, in comparison with other PMU-based protection schemes, is implemented by a lower number of PMUs. Moreover, this backup protection scheme adapts its characteristics to the power system conditions.

The WSCC 9-bus test system and the large-scale IEEE 118-bus power network have been utilized to demonstrate the effectiveness of the proposed backup protection scheme. Various analyzes indicate robustness of the proposed method against different fault locations, different fault types, highimpedance faults and measurement errors. It has also been observed that even if the faulted line is not equipped with any PMUs, the proposed method performs successfully and identifies the faulted line and fault location quickly. Since the proposed scheme provides a closed-form and non-iterative solution for the problem, its operation time does not exceed $403 \mathrm{~ms}$ for all fault conditions. By development of the communication infrastructures and wide area measurement systems (WAMSs) the proposed scheme can be realized in smart transmission grids.

\section{REFERENCES}

[1] A. G. Phadke and J. S. Thorp, Computer Relaying for Power Systems. Hoboken, NJ, USA: Wiley, 2009.

[2] P. M. Anderson, Power System Protection. New York, NY, USA: IEEE Press, 1999.

[3] M. G. Adamiak et al., "Wide area protection-Technology and infrastructures," IEEE Trans. Power Del., vol. 21, no. 2, pp. 601-609, Apr. 2006.

[4] M. Zima, T. Krause, and G. Andersson, "Evaluation of system protection schemes, wide area monitoring and control systems," in Proc. 6th Int. Conf. Adv. Power Syst. Control Oper. Manage., Hong Kong, Nov. 2003, pp. $754-759$.

[5] L. Ramesh, S. P. Chowdhury, and S. Chowdhury, "Wide area monitoring protection and control-A comprehensive application review," in Proc. 10th IET Int. Conf. Develop. Power Syst. Protect. Manage. Change (DPSP), Manchester, U.K., 2010, pp. 1-4.

[6] S. H. Horowitz and A. G. Phadke, "Third zone revisited," IEEE Trans. Power Del., vol. 21, no. 1, pp. 23-29, Jan. 2006.

[7] A. G. Phadke, "Synchronized phasor measurements in power systems," IEEE Comput. Appl. Power, vol. 6, no. 2, pp. 10-15, Apr. 1993.

[8] A. G. Phadke and J. S. Thorp, Synchronized Phasor Measurements and Their Applications. New York, NY, USA: Springer, 2008.

[9] A. G. Phadke and B. Kasztenny, "Synchronized phasor and frequency measurement under transient conditions," IEEE Trans. Power Del., vol. 24, no. 1, pp. 89-95, Jan. 2009.

[10] J. De La Ree, V. Centeno, J. S. Thorp, and A. G. Phadke, "Synchronized phasor measurement applications in power systems," IEEE Trans. Smart Grid, vol. 1, no. 1, pp. 20-27, Jun. 2010.
[11] H. Y. Li et al., "A new type of differential feeder protection relay using the global positioning system for data synchronization," IEEE Trans. Power Del., vol. 12, no. 3, pp. 1090-1099, Jul. 1997.

[12] I. Hall et al., "New line current differential relay using GPS synchronization," in Proc. IEEE Power Tech Conf., vol. 3. Bologna, Italy, Jun. 2003.

[13] J. A. Jiang, L. Chih-Wen, and C. Ching-Shan, "A novel adaptive PMUbased transmission-line relay-design and EMTP simulation results," IEEE Trans. Power Del., vol. 17, no. 4, pp. 930-937, Oct. 2002.

[14] M. M. Eissa, M. E. Masoud, and M. M. M. Elanwar, "A novel back up wide area protection technique for power transmission grids using phasor measurement unit," IEEE Trans. Power Del., vol. 25, no. 1, pp. 270-278, Jan. 2010

[15] J. Ma et al., "A fault steady state component-based wide area backup protection algorithm," IEEE Trans. Smart Grid, vol. 2, no. 3, pp. 468-475, Sep. 2011.

[16] Y. Liao, "Fault location for single-circuit line based on bus-impedance matrix utilizing voltage measurements," IEEE Trans. Power Del., vol. 23, no. 2, pp. 609-617, Apr. 2008.

[17] S. Azizi, A. S. Dobakhshari, S. A. N. Sarmadi, and A. M. Ranjbar, "Optimal PMU placement by an equivalent linear formulation for exhaustive search," IEEE Trans. Smart Grid, vol. 3, no. 1, pp. 174-182, Mar. 2012.

[18] F. Aminifar, A. Khodaei, M. Fotuhi-Firuzabad, and M. Shahidehpour, "Contingency-constrained PMU placement in power networks," IEEE Trans. Power Syst., vol. 25, no. 1, pp. 516-523, Feb. 2010.

[19] H. Saadat, Power System Analysis, 2nd ed. New York, NY, USA: McGraw-Hill, 2002.

[20] J. J. Grainger and W. D. Stevenson, Power System Analysis. New York, NY, USA: McGraw-Hill, 1994

[21] N. Kang and Y. Liao, "Double-circuit transmission-line fault location utilizing synchronized current phasors," IEEE Trans. Power Del., vol. 28 , no. 2, pp. 1040-1047, Apr. 2013.

[22] A. Abur and A. G. Exposito, Power System State Estimation: Theory and Implementation. New York, NY, USA: Marcel Dekker, 2004.

[23] A. J. Wood, B. F. Wollenberg, and G. B. Sheblé, Power Generation, Operation, and Control, 3rd ed. Hoboken, NJ, USA: Wiley, 2014.

[24] R. D. Zimmerman, C. E. Murillo-Sanchez, and D. Gan, MATPOWERA MATLAB Power System Simulation Package. [Online]. Available: http://www.pserc.cornell.edu/matpower

[25] Power Systems Test Case Archive. [Online]. Available: http://www.ee.washington.edu/research/pstca

[26] G. Benmouyal, "Removal of DC-offset in current waveforms using digital mimic filtering," IEEE Trans. Power Del., vol. 10, no. 2, pp. 621-630, Apr. 1995.

[27] B. Naduvathuparambil, M. C. Valenti, and A. Feliachi, "Communication delays in wide area measurement systems," in Proc. 34th Southeast. Symp. Syst. Theory, Huntsville, AL, USA, Mar. 2002, pp. 118-122.

M. Kalantar Neyestanaki (S'14) received the B.Sc. degree in electrical engineering and physics, and the M.Sc. degree in electrical engineering from the Sharif University of Technology, Tehran, Iran, in 2011 and 2013, respectively.

His current research interests include applications of wide-area monitoring, protection and control system in smart grids, digital protective relays, WAMS and SCADA security, and communication technology applied to power systems.

A. M. Ranjbar received the M.Sc. degree from Tehran University, Tehran, Iran, and the Ph.D. degree from Imperial College London, London, U.K., in 1967 and 1975, respectively, both in electrical engineering.

Since 1975, he has been with the Department of Electrical Engineering, Sharif University of Technology, Tehran, where he is currently a Full Professor. He was the Director of Niroo Research Institute, Tehran, from 1996 to 2007. His current research interests include power system protection and operation, electrical machines, and smart grid.

Prof. Ranjbar was the recipient of the Outstanding Faculty Award of the Sharif University of Technology, Tehran in 1996, and the Distinguished Professor Award in the Department of Electrical Engineering in 1998. He was elected as a Memorable Figure in 2004 for 30 years of involvement in both academia and industry. 